\title{
Perceptions de pères vivant avec un fils ou une fille adulte ayant une déficience intellectuelle sur la transition de l'après-parents
}

\author{
Zoé Faubert et Georgette Goupil \\ Département de psychologie, Université du Québec à Montréal
}

\begin{abstract}
With the increase in life expectancy, many people with intellectual disabilities (ID) are living in the family home with their parents. This research focuses on the experience of 17 fathers of adults with ID. These fathers answered a questionnaire including open and closed questions. During the individual interview, fathers described their motivations to cohabit with their son or daughter, cohabitation benefits and constraints, housing options considered and planning for the future. Results indicate that fathers chose this cohabitation. However, they experience anxiety because they do not know who will support the adult with ID when they can no longer do so. Postparental planning considerations include legal concerns and informal discussions with siblings or the extended family. These results describe a complex parental situation in which there is interaction between their emotions, their attachment to the adult with ID and their previous experiences with residential, social or rehabilitation services.
\end{abstract}

\section{RÉSUMÉ}

Avec l'augmentation de la longévité, plusieurs personnes ayant une déficience intellectuelle (DI) vieillissent au domicile familial avec leurs parents. Cette recherche porte sur l'expérience de 17 pères d'adultes ayant une DI. Ces pères ont répondu à un questionnaire incluant des questions ouvertes et fermées en entrevue individuelle afin de décrire leurs motivations pour cohabiter avec leur fils ou leur fille ayant une DI, les bénéfices et contraintes de cette cohabitation, les services résidentiels futurs envisagés et la planification de "l'après-parents ». Les résultats indiquent que les pères ont choisi cette cohabitation. Ils éprouvent de l'anxiété, ignorant qui assumera le soutien auprès de l'adulte avec une DI lorsqu'ils ne pourront plus le faire. Leur planification de l'après-parents correspond à des démarches légales et à des conversations avec la fratrie ou la famille élargie. La discussion souligne la complexité de la situation où entrent en jeu les émotions des parents, leur attachement à l'adulte ayant une DI et les expériences antérieures avec les services résidentiels, sociaux ou de réadaptation.

Manuscript received: / manuscrit reçu : 25/01/17

Manuscript accepted: / manuscrit accepté : 07/12/18

Keywords: postparental period, transition, father, intellectual disability, aging

Mots-clés : après-parents, transition, père, déficience intellectuelle, vieillissement

Correspondence and requests for reprints should be sent to / La correspondance et les demandes de tirés-à-part doivent être adressées à :

Georgette Goupil, Ph.D.

Département de psychologie

Université du Québec à Montréal

CP 8888, Succ. Centre-Ville

Montréal, QC H3C 3P8

La déficience intellectuelle (DI) se caractérise par des limitations significatives $\mathrm{du}$ fonctionnement intellectuel et du comportement adaptatif lequel se manifeste dans les habiletés conceptuelles, sociales et pratiques. Cette incapacité survient avant l'âge de
18 ans (Schalock et al., 2011, p. 6). Selon la métaanalyse internationale de Maulik, Mascarenhas, Mathers, Dua et Saxena (2011), la prévalence de la DI se situe à environ $1 \%$ de la population $(10,37 / 1000$ personnes). 
L'espérance de vie de la population générale et des personnes ayant une DI a augmenté considérablement au cours des dernières années (Azéma \& Martinez, 2005). À titre indicatif, pour l'ensemble de la population québécoise, cette espérance se situe à 82,2 ans, hommes et femmes confondus (Institut de la statistique du Québec, 2015). Selon une étude australienne, la longévité des personnes ayant une DI fluctuerait en fonction des causes et du degré de déficience: déficience légère 74 ans; déficience moyenne 67,6 ans; déficience sévère 58,6 ans (Bittles, Petterson, Sullivan, Hussain, Glasson \& Montgomery, 2002). Avec cette augmentation de l'espérance de vie et avec la désinstitutionalisation entrainant le maintien dans le milieu de vie naturel, de nombreux parents d'un adulte ayant une DI vieillissent en parallèle avec cet adulte au domicile familial (Azéma et Martinez, 2005; Burke et Heller, 2016; Gouvernement du Canada, 2004; Rioux et Saint-Pierre, 2013).

Plusieurs personnes ayant une DI, particulièrement celles présentant des déficiences moyenne, sévère ou profonde, ont besoin de soutien toute leur vie. La famille, c'est-à-dire les parents et la fratrie, apportent cette assistance dans la majorité des situations (Taggart, Truesdale-Kennedy, Ryan \& McConkey, 2012). Ces parents rapportent cependant des problèmes de santé et des niveaux élevés d'anxiété (Seltzer, Floyd, Song, Greenberg \& Hong, 2011; Taggart et al., 2012). L'âge, les problèmes de santé et un désir d'indépendance des parents représentent, selon Grey, Griffith, Totsika et Hastings (2015), les principaux motifs pour chercher un autre lieu résidentiel pour l'adulte ayant une DI.

Les transitions représentent les passages entre les périodes de vie (exemple : la transition de l'école vers la vie adulte) ou encore les changements significatifs d'un lieu de vie à un autre (Goupil, 2004). Parmi ces transitions, l'après-parents implique des démarches et de multiples changements pour la personne ayant une DI et sa famille. De nombreux parents doivent inscrire leur fils ou leur fille adultes sur de longues listes d'attente pour obtenir des ressources résidentielles (Burke et Heller, 2016; Rioux et St-Pierre, 2013). En changeant de lieu d'hébergement, la personne ayant une DI verra son réseau social et ses habitudes modifiées (Longtin, 2015). Elle devra s'adapter aux nouvelles règles de vie de la ressource résidentielle. De plus, la famille et la personne avec DI se voient confrontées à une rupture non seulement physique mais aussi affective (Azéma et Martinez, 2005). Les parents assumant pour la majorité des adultes ayant une DI le soutien requis (Ouellette-Kuntz et al., 2005), la transition implique éventuellement le choix d'une ou de plusieurs personnes qui assumeront un suivi lorsque les parents ne seront plus en mesure de le faire.
Afin d'éviter les situations de crise, des organismes communautaires recommandent une planification à long terme impliquant la personne ayant une DI et sa famille (Ministère des Services sociaux et communautaires de l'Ontario, 2013). Si la planification de l'aprèsparents est reconnue essentielle, des études font état des réserves des parents à l'effectuer, entre autres, à cause de leur attachement à la personne ayant une DI, des avantages associés à la cohabitation et des difficultés à obtenir les services (Bernard \& Goupil, 2012; Taggart et al., 2012). Ainsi, les services sociaux et de santé doivent répondre à des demandes urgentes suite à la maladie ou au décès des parents. Les personnes ayant une DI peuvent alors être orientées vers les services résidentiels disponibles et non vers ceux convenant le mieux à leurs besoins. Les services sociaux et de réadaptation sont aussi confrontés aux longues listes d'attente pour une résidence et aux besoins de soutien criants des familles (Woodman, Mallick, Anderson, \& Esbensen, 2014).

L'après-parents ayant avantage à être planifié, il importe de connaître les attitudes et les motivations des parents à cet égard. Or, seules quelques études canadiennes ont évalué cette transition des personnes qui ont une DI (Bernard \& Goupil, 2012; Joffres, 2002; LacasseBédard, 2009). Des expériences négatives des parents auprès des services, une vision pessimiste des services résidentiels, un quotidien sans difficulté majeure et des valeurs familiales justifient le maintien de la personne ayant une DI dans sa famille (Bernard \& Goupil, 2012; Lacasse-Bédard, 2009). Parmi les quelques études québécoises disponibles, Bernard et Goupil (2012) ont évalué les perceptions de l'après-parents de 12 mères québécoises ( $M_{\text {âge }}=65,1$ ans) d'un fils ou d'une fille ayant une DI de plus de 18 ans ( $M_{\text {agge }}=36,9$ ans) et habitant au domicile familial. Six mères ont utilisé des services résidentiels autres que le domicile pour leur fils ou leur fille. Toutefois, insatisfaites de la réponse donnée par ces milieux aux besoins de leur fils ou de leur fille ayant une DI, elles les ont réintégrés dans le foyer familial. Même si ces mères avaient en moyenne 65 ans, leur planification de l'après-parents se révélait peu avancée. Elles percevaient des avantages à la cohabitation tels une vie agréable et un meilleur respect des valeurs humaines. Toutefois, face à l'avenir, elles ressentaient surtout de l'inquiétude, de l'anxiété et de la peur.

Rapportant l'étude australienne de Bigby (1996), Bigby, Webber et Bowers (2015) indiquent que près de la moitié des personnes ayant une DI $(N=62)$ vivent, après le décès de leurs parents, avec la fratrie. Cet arrangement $\mathrm{n}^{\prime}$ a toutefois qu'un caractère temporaire, car la plupart des personnes ayant une DI habitent, en vieillissant, dans une autre ressource d'hébergement que la résidence de la fratrie (Heller \& Kramer, 2009). 
Les frères et les sœurs jouent cependant un rôle majeur dans la supervision des services (Bigby et al., 2015). Villeneuve, Goupil et Gascon (2012) ont décrit les rôles perçus de 13 fratries québécoises adultes $\left(M_{\hat{a} g e}=26,34\right.$ ans) d'une personne ayant une DI. Toutes ces fratries souhaitaient s'engager, sur divers plans, dans le soutien auprès de la personne ayant une DI.

La plupart des études internationales concernent les perceptions des mères ou de couples de parents. En effet, peu d'études ont évalué les perceptions des pères dans le contexte de l'après-parents (Davys, Mitchell, \& Martin, 2017). Dans une recension sur les pères d'enfants ayant une DI (entre la naissance et 22 ans), Davys, Mitchell et Martin (2017) concluent que, si dans certaines situations, le père joue une fonction centrale dans le soutien à son enfant, en général, la mère assume cette aide. Cette recension soulève aussi les résultats contradictoires, à la fois positifs et négatifs, sur la qualité de vie et la satisfaction conjugale des pères. Davys et al. soulignent aussi la nécessité d'effectuer d'autres études particulièrement auprès de pères d'adultes ayant une DI. Une recension sur les principales banques de données confirme le peu d'études canadiennes sur l'expérience rapportée des pères auprès d'un adulte ayant une DI et la planification de la transition vers l'après-parents.

\section{Buts et objectifs de l'étude}

Cette étude exploratoire, dans la continuité de celle de Bernard (2011), vise à mieux connaitre les actions et l'expérience rapportées de pères d'adultes ayant une DI et vivant au domicile familial. Elle a pour objectifs de: 1) décrire les avantages et les contraintes rapportés par les pères qui cohabitent avec leur enfant ayant une DI à l'âge adulte; 2) explorer les émotions et les rêves des pères face à l'avenir de l'adulte ayant une DI; 3) décrire les actions effectuées pour planifier l'après-parents, dont les actions en regard des services résidentiels ainsi que le rôle confié à la fratrie dans la planification de l'avenir de la personne ayant une DI.

\section{Méthode}

\section{Participants}

Dix-sept pères biologiques âgés entre 49 à 70 ans $(M=$ $61, M d=62)$ ont participé à l'étude. Ils habitent à temps plein avec leur fils ou leur fille ayant une DI $(N=17)$, âgés entre 21 et 36 ans $(M=29)$, dans des familles biparentales avec les mères biologiques, sauf dans un cas. Huit pères ont un emploi, deux sont en retraite progressive et sept sont à la retraite. Pour que ces pères soient inclus dans l'étude, leurs fils ou leurs filles devaient habiter au domicile familial, présenter une DI moyenne, sévère ou profonde et avoir plus de 18 ans. Ces adultes ayant une DI n'ont toutefois pas été interviewés. Le tableau 1 décrit les caractéristiques des fils et des filles des pères participants.

\section{Instruments}

Deux instruments ont été utilisés, une fiche de renseignements personnels et le Questionnaire sur l'aprèsparents - version pour les pères est adapté de Bernard (2011). Ce questionnaire inclut des questions fermées et ouvertes. Les questions fermées permettent d'obtenir des informations précises (p. ex., avez-vous déjà envisagé d'autres lieux de vie?) alors que les questions ouvertes (p. ex., quels sont vos rêves pour votre enfant?) laissent aux pères l'occasion de décrire leur situation ou leurs émotions. Nous avons retenu, pour le présent article, les questions permettant de répondre aux buts de l'étude : motifs et expériences en regard de la cohabitation, rêves et émotions face à l'avenir et planification de l'après-parents. Le questionnaire de Bernard (2011) a fait l'objet d'une validation de contenu par cinq experts et $\mathrm{d}$ 'une expérimentation auprès de 12 mères d'adultes ayant une DI. Nous avons pris en compte les recommandations de Bernard suite à son expérimentation pour bonifier le questionnaire ainsi qu'adapter le questionnaire pour des pères. Nous avons effectué une nouvelle évaluation du contenu par cinq experts. Ces derniers se sont prononcés sur le contenu et la forme des questions en fonction des objectifs de l'étude. Ils ont proposé des changements sur les termes utilisés, l'ordre et la formulation des questions. Enfin, la version révisée a été pré-expérimentée auprès de deux pères.

Tableau 1 : Caractéristiques des fils et des filles ayant une DI $(\mathrm{N}=17)$ des pères participants

\begin{tabular}{lr}
\hline Caractéristiques des fils ou filles & $\mathbf{n}$ \\
\hline Hommes & 9 \\
Femmes & 8 \\
Niveau de déficience & 11 \\
Moyenne & 5 \\
Sévère & 1 \\
Profonde & 9 \\
Syndrome génétique associé (p. ex., trisomie) & \\
Langage & 12 \\
Verbal & 5 \\
Non-verbal (ne parle pas) & 16 \\
Présence d'une fratrie & 1 \\
Enfant unique & 13 \\
Présence d'un emploi & \\
Région québécoise de provenance & 10 \\
Montérégie & 4 \\
Saguenay-Lac-Saint-Jean & 2 \\
Montréal & 1 \\
Laval &
\end{tabular}




\section{Procédure et considérations éthiques}

Nous avons choisi d'administrer oralement le questionnaire en entrevue afin de ne pas limiter, par l'écriture, la longueur des réponses des participants. Les entrevues se déroulent, selon le choix des pères, à leur domicile ou dans un bureau de l'université. Les entrevues enregistrées numériquement varient entre 45 minutes et 1 heure $45\left(M_{\text {durée }}=80\right.$ minutes $)$. Cette variabilité s'explique par le fait que certains participants s'exprimaient davantage que d'autres. Afin de garder la cohérence d'une entrevue à l'autre, la première auteure a effectué toutes les entrevues.

Le comité d'éthique du département de psychologie de l'Université du Québec à Montréal ainsi que le comité d'éthique conjoint des Centres de réadaptation en déficience intellectuelle et en trouble du spectre de l'autisme ont approuvé ce projet. Les pères ont signé un formulaire de consentement. Ils étaient libres de ne pas répondre à certaines questions, c'est pourquoi, dans les résultats, le nombre de répondants fluctue selon les questions.

\section{Analyse des données}

Les questions fermées de la fiche de renseignements personnels et du Questionnaire sur l'après-parents version pour les pères sont analysées en fréquences. La méthode de Miles et Huberman (2003) a permis l'analyse de contenu des questions ouvertes. Toutes les entrevues ont d'abord été transcrites intégralement en ayant pris soin d'enlever tout renseignement identifiant un participant.

Nous avons choisi d'analyser manuellement le contenu des entretiens, car plusieurs verbatim exigeaient des déductions du chercheur (expressions variées en fonction des régions, référence à des services locaux, etc.). Après plusieurs lectures du verbatim par la première auteure, les segments de réponses furent identifiés pour ensuite être regroupés par thèmes. Ces thèmes ont été organisés dans une première grille d'analyse de contenu dans laquelle le verbatim a été classifié. Une assistante de recherche a, par la suite, classé le verbatim, de manière indépendante, en utilisant aussi cette première grille. Ces deux analyses indépendantes ont été comparées à l'aide du Kappa de Cohen. Les catégories de la grille n'atteignant pas un coefficient d'accord de 0,80 ont été discutées et précisées par les deux personnes ayant effectué cette première analyse.

Suite aux modifications de la grille, la première auteure et l'assistante de recherche ont de nouveau analysé les données de manière indépendante. Le Kappa de Cohen indique, entre ces deux analyses, des coefficients de fidélité variant entre 0,80 et 1,00.

\section{Résultats}

Le tableau 2 présente les questions de l'entretien et les thèmes de réponses ressortis de l'analyse. Ces thèmes sont présentés en ordre décroissant selon le nombre de pères les ayant mentionnés.

\section{Motifs pour maintenir l'adulte ayant une DI au domicile familial}

À la question "votre enfant vit actuellement avec vous, est-ce votre choix? ", tous les pères disent valoriser la cohabitation car elle va de soi et se révèle agréable. Les pères disent que la responsabilité de maintenir leur fils ou leur fille au domicile familial leur revient. Ils justifient aussi ce choix par les liens affectifs avec leur fils ou leur fille. Ils ne regrettent pas cette décision. Des pères désirent vivre auprès de l'adulte ayant une DI « jusqu'au bout » c'est-àdire tant que leur état de santé et leur autonomie permettront d'assumer ce soutien. Un père retraité souhaite cette cohabitation pour compenser son absence au moment où il travaillait.

\begin{abstract}
On n'a jamais pensé que notre fille ce n'était pas une personne à part entière dans notre famille. On n'a jamais vraiment pensé à ça (père 7 : fille de 32 ans).

C'est notre fille, et on n'est pas pour la lâcher dans la nature, n'importe où (père 16 : fille de 35 ans).

$C^{\prime}$ est nos obligations et on les assume (père 10 : fille de 30 ans).

Quand j'ai décidé de prendre ma pension, bien j'ai dit "je vais avoir du temps, donc je vais compenser pour le temps où je n'ai pas été là" (père 6 : fille de 36 ans).
\end{abstract}

\section{Apports de la cohabitation}

Treize des quinze participants ayant répondu sur les apports de la cohabitation en perçoivent des avantages alors que deux n'en voient aucun. Des participants soulignent le renforcement du lien familial ainsi que le bonheur et la vie dans le domicile parental.

\footnotetext{
$C^{\prime}$ est sûr qu'adulte c'est différent. On a eu beaucoup de peine, mais on a eu beaucoup de joie avec elle. On a encore des joies avec elle, et il n'est pas question qu'on la place au moment où on se parle (père 13 : fille de 27 ans).

$C^{\prime}$ 'est une folie, peut-être, mais c'est mon poteau de vieillesse. Elle est partie une journée, et j'ai trouvé ça dur, plate. C'était vide dans la maison (père 5 : fille de 34 ans).
}

Les pères vivent davantage le moment présent, profitent d'une cohabitation agréable et développent des valeurs humaines telles l'écoute, la tolérance, la patience et la sensibilité envers l'autre. 
Tableau 2 : Fréquences des thèmes rapportés par les pères aux questions de l'entretien

Questions

Apports de la cohabitation

Contraintes de la cohabitation

Autres lieux de vie envisagés

Émotions face à l'avenir

Rêves des pères pour la personne ayant une DI

Planification de l'après-parent

Relation et soutien de la fratrie

\section{Thèmes}

Cela va de soi

Cohabitation agréable

Désir d'accompagner la personne ayant une DI jusqu'au bout

Amour envers la personne ayant une DI

Vie agréable au domicile

Développement de valeurs humaines

Renforcement du lien familial

Centration sur l'instant présent

Sentiments de la personne ayant une DI à l'égard de ses parents

Aucun apport

Difficultés organisationnelles (p. ex., horaires, voyages, loisirs)

Conséquences sur la carrière

Difficultés dans les sorties liées aux problèmes de comportement

Manque d'intimité du couple

Aucune contrainte

Domicile actuel modifié

Établissement public

Autres lieux

Aucun autre lieu

Anxiété

Confiance en l'avenir

Sentiment d'injustice

Incertitude

Grande tristesse

Aucune émotion

Être heureux et bien vivre

Trouver un milieu de vie avec une bonne qualité de vie

Souhaiter que la personne ayant une DI décède avant le parent

Être capable de se débrouiller seul

Être bien entouré

Occuper un emploi

S'accepter

Aucun rêve

n

10

4

2

2

5

5

3

2

2

10

2

2

2

3

4

3

3

7

5

1

1

2

1

3

Testament, fiducie ou curatelle

Entente informelle avec la fratrie

Liste d'attente pour une autre résidence

Épargne

Entente informelle avec famille élargie

Absence de démarche

5

4

4

3

2

2

0

11

9

2

2

1

Type de relation

Relation positive

Relation minimale

Relation tendue

Degré d'engagement

Minimal

Assistance informelle

Aide formelle (p. ex., gardiennage, bains)

Entente prévue

Aspect financier

Autre lieu de résidence avec supervision de la fratrie

Autre lieu de résidence sans supervision de la fratrie

Garde totale

Refuse l'aide de la fratrie

Aucune entente 
Tableau 2: Continued

\begin{tabular}{ll}
\hline Questions & Thèmes \\
\hline Discussion avec la personne ayant une DI sur son avenir & Présence de la discussion \\
Oui & Non \\
Contenu de la discussion & Avenir résidentiel \\
8 & Décès éventuel du parent \\
& Changement de résidence \\
& Avenir professionnel \\
& Avenir amoureux \\
& Différences causées par la DI entre la personne et les autres personnes \\
& Motifs justifiant l'absence de discussion \\
& Adulte ayant une DI ne comprend pas la notion du temps \\
& Parent non prêt à cette discussion \\
& Personne ayant une Dl jugée comme non prête \\
& Personne ayant une Dl jugée non intéressée à son avenir \\
& Craintes des inquiétudes de la personne ayant une DI \\
\hline
\end{tabular}

\section{Contraintes de la cohabitation}

Nous avons demandé aux pères s'il y avait des contraintes à la cohabitation et, le cas échéant, leur nature. Des seize pères répondant à la question, trois ne voient pas de désavantages à la cohabitation. Les treize autres décrivent des difficultés sur le plan de l'organisation quotidienne. Ainsi, ils doivent tenir compte des rendez-vous avec les services requis par la personne ayant une DI. De plus, le domicile familial doit se situer à proximité de ces services (p. ex., centre de réadaptation en déficience intellectuelle). Des pères mentionnent la perte de liberté dans les voyages, les loisirs ou des difficultés dans leurs propres relations sociales. Ils perçoivent un manque de services. Cette lacune concerne, entre autres, des services de répit. Les pères souhaitent que ces services prennent en compte leurs dates de vacances. La cohabitation a aussi des conséquences sur la carrière telles une retraite prématurée ou l'impossibilité d'accepter une promotion professionnelle. Certains pères craignent les sorties avec la personne ayant une DI à cause de comportements difficiles en public. Enfin, deux participants mentionnent avoir peu l'occasion d'être seuls en couple. Ils soulignent devoir toujours partager leurs activités avec la personne ayant une DI.

Ce qui est difficile, par exemple, s'il y a une mortalité dans la famille, moi et ma conjointe ne pouvons y aller ensemble. Parce qu'on n'a pas de gardien immédiat (père 4 : fils de 28 ans).

On aimerait voyager peut-être un petit peu plus, parce qu'on est rendu quasiment à la limite du voyage à notre âge (père 6 : fille de 36 ans).

Son maniérisme fait que moi, je suis cloué à la maison. Je ne peux pas aller nulle part (père 13: fille de 27 ans).
On (père et mère) n'est jamais seuls. Regarde, moi je ne démords pas là-dessus, on n'est jamais tout seul... On n'est jamais tout seul (père 17 : fils de 26 ans).

\section{Autres lieux de résidence envisagés}

Dix pères ont envisagé d'autres lieux d'hébergement que le foyer parental pour la personne ayant une DI. Des pères souhaitent utiliser une ressource non institutionnelle faisant partie des services publics d'adaptation et de réadaptation en déficience intellectuelle, telles les ressources de type familial ou les ressources intermédiaires de type maison d'accueil. Certains autres participants veulent modifier leur domicile en l'agrandissant ou en le transformant en une résidence intergénérationnelle.

\footnotetext{
Ce qu'on aimerait peut-être ici, c'est d'agrandir. Que [nom du fils] ne soit pas toujours confiné dans le sous-sol (...). Eł qu'il ait peut-être aussi sa porte d'entrée (père 9 : fils de 23 ans).
}

Sept participants, n'ayant pas envisagé d'autres options, ne sont pas prêts à choisir une autre résidence que celle de la famille. Plusieurs ne sont pas satisfaits des ressources disponibles. Certains pères disent avoir peu confiance aux administrateurs des ressources résidentielles et ne sont pas encore préparés à y loger leur fils ou leur fille ayant une DI.

J'aime mieux me priver à l'os que de l'envoyer dans une famille d'accueil, non je ne serais pas capable. Non : je vais vendre ma maison, je vais vendre mon chalet, je vais rester dans un $2 \frac{1}{2} s^{\prime} i l$ le faut. Je vais payer quelqu'un pour s'occuper de mon gars (père 17 : fils de 26 ans).

\section{Émotions face à l'avenir}

Les 15 pères ayant décrit leurs émotions ressentent, face à l'avenir, des émotions surtout négatives soit de 
l'anxiété, du stress ou de la peur. Ils se demandent « qui » prendra soin de l'adulte ayant une DI lorsqu'ils ne seront plus en mesure de le faire. Certains pères anticipent avoir de la peine lorsque la personne ayant une DI quittera le domicile familial les privant ainsi de moments plaisants associés à la vie commune actuelle.

\begin{abstract}
Moi, je ne voudrais pas qu'elle parte jamais d'ici en fait. Mais de penser qu'un jour, elle va partir, là ça me fait de la peine (père 7 : fille de 32 ans).
\end{abstract}

Un participant doute de la capacité des autres personnes de discerner les sentiments de son fils, car ce dernier communique de manière non verbale. Un père dit ressentir une grande tristesse. Certains résument leurs émotions par une seule expression alors que d'autres s'expriment plusieurs minutes sur le sujet.

Un père fait confiance en l'avenir parce que son fils ayant une DI est capable d'apprentissage et de conserver ce qu'il apprend. A contrario, trois pères, parmi les plus jeunes de l'étude, disent ne rien ressentir face à l'avenir.

Moi, ça me laisse vraiment indifférent. Je n'anticipe pas le fait que je vais vieillir et que lui va vieillir (père 9 : fils de 23 ans).

Je ne sais pas si c'est à cause de son état de santé, mais nous on a toujours pris ça au jour le jour (père 14 : fils de 26 ans).

L'avenir? Pas grande émotion, là (père 17 : fils de 26 ans).

\section{Rêves des pères pour leur fils ou leur fille ayant une DI}

Ces rêves visent surtout le bonheur, la qualité de vie, le soutien et l'autonomie de l'adulte ayant une DI. Aucun rêve n'est mentionné concernant des espoirs où la personne ayant une DI fonde une famille, vive en couple ou donne naissance à des enfants.

Mes rêves, c'est que je sache qu'il y ait toujours quelqu'un qui puisse jeter un regard pour le protéger (père 11 : fils de 34 ans).

On pourrait dire que s'il est heureux, c'est le plus important. S'il est heureux avec nous autres tant mieux, s'il est heureux avec d'autres, ça ferait. S'il $\mathrm{n}^{\prime}$ est pas malheureux, parce qu'à date, il a des amis, lui il est heureux (père 15 : fils de 22 ans).

J'espère qu'il aura toujours l'occasion d'être dans un milieu où il aura une qualité de vie que ce soit chez nous, que ce soit dans un foyer de groupe, ou que ce soit ailleurs. Mais une qualité de vie qu'il va apprécier (père 9 : fils de 23 ans).

Bien nous autres, on veut que notre enfant devienne le plus autonome possible pour être capable de se débrouiller dans le monde dans lequel on vit. Lui donner des outils pour qu'elle puisse être capable de vivre en société (père 7 : fille de 32 ans).
Quatre pères disent souhaiter que l'adulte ayant une DI les précède dans la mort.

Le plus beau rêve, je sais que ce n'est pas correct, mais c'est qu'on ait le temps de l'enterrer avant qu'on parte (père 4 : fils de 28 ans).

Si je pouvais lui survivre, ce serait l'idéal (père 11 : fils de 34 ans).

\section{Planification de l'après-parents}

Des questions concernent les actions en regard du moment où les pères ne seront plus en mesure $\mathrm{d}$ 'assurer leur soutien à la personne ayant une DI. Quinze pères disent avoir effectué des démarches concrètes, alors que deux n'en ont fait aucune. Onze pères ont planifié les aspects légaux par un testament, une fiducie ou une curatelle (c.-à-d., régime de protection pour un adulte inapte à faire valoir ses droits ou administrer ses biens). Neuf ont pris des ententes, lors de discussions, avec la fratrie. Ces ententes toutefois ne sont pas formalisées légalement. Ainsi, certains pères se sont entendus, avec la fratrie, sur une supervision de l'adulte ayant une DI qui demeurera alors dans un milieu de vie (ou réadaptation) substitut. D'autres ont épargné de l'argent pour mieux répondre aux besoins de la personne ayant une DI (p. ex., pour des besoins précis liés à l'adulte ou pour des loisirs). Pour deux pères (67 et 70 ans), parmi les plus âgés des participants, la démarche consiste dans l'inscription sur une liste $\mathrm{d}^{\prime}$ attente pour un autre milieu de vie.

La liste d'attente, mais qui peut attendre jusqu'à 5 ans? Il faut attendre qu'il y ait une place qui se libère. Et faut-il encore que la place soit rapprochée de la maison. S'ils vous disent que c'est à Chicoutimi, on ne peut pas aller la voir toutes les fins de semaine (père 16 : fille de 35 ans).

Comme j'ai dit à mon deuxième, " tu t'organises, pour l'organiser ». II m'a dit «papa, ne t'inquiète pas, je vais $\mathrm{m}^{\prime}$ en occuper. » Mais ça advienne que pourra, moi je vais être mort. (...) Je ne pourrai plus faire grand-chose (père 12 : fille de 33 ans).

Deux pères n'ont fait aucune démarche. Le premier ne se sent pas capable d'obliger une personne à soutenir l'adulte ayant une DI. Le deuxième dit vivre selon la volonté de Dieu:

Non, je ne pense pas à ça. Moi, je vis toujours seulement à la volonté de Dieu. Je suis d'accord, si le Dieu veut. (...) Pour moi ça se passe très bien (père 8 : fils de 22 ans).

\section{Soutien de la fratrie}

Les frères et les sœurs représentent, dans plusieurs familles, la relève des parents. Dans ce contexte, les pères sont d'abord questionnés sur la relation entre la fratrie et la personne ayant une DI. Les relations 
diffèrent d'une famille à l'autre de même que le degré d'engagement souhaité par les fratries. Ce niveau d'engagement varie entre des appels téléphoniques ponctuels et une aide formelle et fréquente.

La relation ça commence un petit peu là se développer), elle (sa sœur) a 25 ans, elle est rendue à Montréal. Parfois, il l'appelle, mais (la relation) c'est juste cela, pas trop encore (père 3 : fils de 21 ans).

Souvent, elles (ses sœurs) vont être là, quand on n'est pas là. Donc, elles vont s'assurer qu'il est correct, qu'il est rentré de l'école (père 9 : fils de 23 ans).

Quinze pères ont répondu à la question sur les discussions avec la fratrie au sujet de l'avenir de l'adulte ayant une DI. Ces discussions ont concerné, pour six pères, les aspects financiers, pour trois pères, les services résidentiels avec suivi de la fratrie et pour deux autres, ces services sans suivi de la fratrie. Deux pères ont abordé la responsabilité de la garde totale de l'adulte ayant une DI par la fratrie. Un père a refusé l'aide de la fratrie sous prétexte d'une lourde responsabilité et pour un dernier père, il n'y a eu aucune entente.

Elle, sa sœur, son inquiétude c'était, elle avait peutêtre peur qu'on lui dise : regarde, tu vas le garder. Je lui ai dit: je ne veux pas que tu le gardes, je veux que tu le places dans un endroit où il va être bien (père 3 : fils de 21 ans).

Avec mes filles, on disait en riant: il ne va pas pouvoir rester chez vous plus tard, parce que peutêtre que votre mari n'en voudra pas. Bien, moi je ne veux pas de mon mari à ce moment-là (père 9 : fils de 23 ans).

\section{Communications avec la personne ayant une DI sur son avenir}

Dans une perspective d'autodétermination, la personne ayant une DI doit être impliquée dans la planification de l'après-parents avec des moyens facilitant la communication sur cette transition. Quatorze participants ont donc répondu à la question : avez-vous abordé avec votre enfant son avenir? La question ayant suscité beaucoup d'émotions, trois pères n'y ont pas répondu.

Six pères ont eu une discussion sur l'après-parents avec l'adulte ayant une DI. Le contenu portait sur les services résidentiels, le décès éventuel du parent, les différences causées par la DI ainsi que l'avenir amoureux ou au travail.

On lui dit qu'on ne sera pas toujours là (père 7 : fille de 32 ans).

Mais il est conscient de ça. Que quand on ne sera pas là, il y aura d'autres alternatives pour lui. Ça, il le sait. On lui en parle régulièrement (père 11 : fils de 34 ans).
Tu sais, des fois, on va lui demander : "Qu'estce que tu veux faire plus tard? As-tu le goût de te marier? As-tu le goût d'aller en appartement? Qu'est-ce que tu veux faire comme travail? » Alors, on en discute avec lui (père 9 : fils de 23 ans).

Huit pères n'ont pas discuté de l'avenir, croyant que l'adulte ayant une DI ne comprend pas la notion du temps. Certains estiment qu'eux ou l'adulte ayant une DI ne sont pas prêts à cette discussion ou encore que l'adulte ayant une DI ne s'intéresse pas à son avenir. Enfin, un père évite cette conversation pour ne pas susciter d'inquiétudes à l'adulte ayant une DI.

Tu connais ton monde, quand tu ouvres des portes, $c^{\prime}$ est que tu veux que ça aille par là. Je n'ouvre pas de portes parce que je ne veux qu'il s'en aille par là tout de suite, je ne veux pas, j'aurais peur de créer un traumatisme, c'est fort (père 17 : fils de 26 ans).

\section{Discussion}

\section{La cohabitation: avantages et contraintes}

Les pères ont choisi la cohabitation et ils y perçoivent des avantages tels avoir de la vie dans la maison, le renforcement du lien familial et le développement de valeurs humaines. Le quotidien sans difficulté majeure justifie, partiellement du moins, dans les familles l'absence de planification de l'après-parents (LacasseBédard, 2009). Les pères décrivent néanmoins de nombreuses contraintes à la cohabitation telles la nécessité de toujours prévoir un soutien pour l'adulte ayant une DI, la planification des horaires, la perte de la liberté personnelle et les restrictions dans l'avancement professionnel. À ces contraintes, s'ajoute pour le couple, l'exigence de partager ses activités avec l'adulte qui a une DI. En effet, les services de répit et de soutien manquent aux participants. La recension de Rioux et StPierre (2013) précise que ces services de répit et soutien sont parmi ceux les plus demandés par les familles. Ces besoins sont peu comblés même s'il s'agit de "la pierre angulaire de la cohabitation » (p.110). Seltzer et al. (2011) soulignent la perte de liberté dans les activités quotidiennes des parents qui doivent répondre aux besoins de soutien de leur fils ou de leur fille. Les contraintes de la cohabitation peuvent également entrainer pour ces parents de la détresse et de l'anxiété (Robinson, Weiss, Lunsky \& Ouellette-Kuntz, 2015). Néanmoins, les pères se montrent attachés à l'adulte ayant une DI par les liens familiaux, et ce, pour la vie entière.

La littérature souligne que des parents préfèrent maintenir la cohabitation afin de prévenir les effets néfastes d'un changement de lieu de résidence (Shaw, Cartwright \& Craig, 2011). Hole, Stainton et Wilson (2013) avancent que la fatigue des parents entraverait la planification de la transition qui exige des démarches entrainant elles aussi de l'épuisement. Les parents ont 
de la difficulté, voire une profonde peine, à imaginer le futur sans leur fils ou leur fille dans le foyer familial. Le départ, dans une ressource résidentielle, de la personne ayant une DI évoque aussi pour ses parents leur propre perte d'autonomie ainsi que leur décès, une conséquence inéluctable du vieillissement (Taggart et al., 2012). Cette absence de planification est probablement aussi influencée par les listes d'attente pour les services résidentiels (Burke et Heller, 2016). La transition de l'après-parents se révèle donc une situation complexe où s'entrecroisent de nombreux facteurs tels les émotions des parents, leur attachement à l'adulte ayant une DI, la méconnaissance ou le manque de services ainsi que des expériences négatives vécues par les parents.

\section{Les options résidentielles autres que le foyer familial}

Wolfensberger (2011) souligne que les personnes ayant une DI utiliseront de plus en plus les ressources résidentielles dans la communauté. Ainsi, la majorité des participants de notre étude anticipent aussi faire ce choix. Toutefois, les démarches de planification sont peu avancées. En outre, certains participants n'ont pas réfléchi aux ressources résidentielles parce qu'ils ne sont pas préparés à la transition, ou encore parce qu'ils se méfient du réseau public, une observation faite également par Taggart et al. (2012). Rioux et Saint-Pierre (2013) relèvent aussi cette réticence des parents à planifier l'avenir résidentiel de l'adulte en situation de handicap. Considérant que le domicile familial est la seule option d'hébergement, les parents gardent donc l'adulte vieillissant dans leur résidence (Bernard \& Goupil, 2012; Krauss \& Seltzer, 1998). Rioux et Saint-Pierre suggèrent d'ailleurs, pour encourager la planification résidentielle, de bonifier le partenariat entre les services sociaux et les ressources d'hébergement.

\section{Émotions face à l'avenir}

Tel qu'observé dans la littérature, les pères ressentent de l'anxiété face au futur ; toutefois, la plupart des études portent sur les mères (Bernard \& Goupil, 2012; Hole, Stainton, \& Wilson, 2013). Taggart et al. (2012) rapportent aussi que la majorité des familles vivent des problèmes liés au rôle d'aidant naturel. Un parent anxieux, déprimé ou ayant des problèmes de santé physique est possiblement moins disposé à planifier le futur. Les difficultés s'amplifient si la DI s'accompagne d'incapacités nécessitant un soutien intense. Trois participants mentionnent ne pas ressentir d'émotions face à l'avenir. Des parents peuvent aussi vivre du déni face à une séparation inéluctable ou adopter une attitude qui leur permet d'éviter trop d'émotions.

\section{Rêves face à l'avenir}

Les pères décrivent leurs rêves centrés surtout sur le bonheur et la qualité de vie de l'adulte ayant une DI tout comme l'ont observé Bernard et Goupil (2012) auprès de mères. L'Enquête sur la participation et les limitations d'activités (Statistique Canada, 2010) présente ce rêve comme réaliste. En effet, la majorité des personnes de plus de 15 ans ayant une incapacité sont habituellement heureuses et intéressées à vivre. Quatre participants entretiennent le rêve que l'adulte ayant une DI les précède dans la mort. Plusieurs autres études ont fait ce constat (Bernard \& Goupil, 2012; Salvatori, Tremblay, \& Tryssenaar, 2003; Taggart et al., 2012). Dans tous ces rêves rapportés par les pères, peu concernent la vie de couple de leur fils ou de leur fille ayant une DI ou la naissance d'enfants, laissant supposer que certains sujets sont encore tabous ou peu abordés dans les familles.

\section{Démarches face à l'avenir}

Tel que mentionné, seulement deux pères, âgés de 67 et 70 ans, ont dit avoir inscrit l'adulte ayant une DI sur une liste d'attente pour un autre lieu résidentiel que le domicile familial. Les démarches des participants concernent surtout des aspects légaux ponctuels (testament, identification d'un tuteur) ou des discussions informelles avec la fratrie et la famille élargie. La littérature scientifique souligne cette imprécision dans la planification qui repose surtout sur le choix d'un tuteur pouvant assumer la responsabilité légale du fils ou de la fille ayant une DI (Bigby, 1996; Hole, Stainton, \& Wilson, 2013). Or, une transition harmonieuse exige des démarches à long terme. Il ne s'agit pas que du moment où la personne ayant une DI quitte le domicile familial, mais d'un ensemble de démarches avant et après ce départ.

Un autre facteur entravant la transition réside dans les listes d'attente pour les services résidentiels (Burke \& Heller, 2016; Woodman, Mallick, Anderson, \& Esbensen, 2014). De plus, les ressources résidentielles disponibles ne répondent pas nécessairement aux besoins et aux préférences de la personne ayant une DI. Cette dernière peut aussi éprouver des émotions négatives suite au changement surtout si cette transition n'a pas été préparée en ayant pris soin de l'impliquer (Longtin, 2015).

\section{Le soutien de la fratrie}

L'engagement de la fratrie varie grandement dans les familles de nos participants. En effet, certains frères et sœurs ne s'engagent pas du tout auprès de l'adulte ayant une DI. D'autres fratries aident quotidiennement les parents, une polarité dans les engagements se 
retrouvant aussi dans la littérature scientifique (Bernard \& Goupil, 2012; Hole et al., 2013). Malgré une relation parfois difficile entre leurs enfants, presque tous les pères mentionnent $s$ 'en remettre à la fratrie pour l'avenir. Des études établissent que les frères et les sœurs deviennent des ressources importantes pour les familles (Davys, Mitchell, \& Haigh, 2010), mais que lorsque les parents décèdent, ce soutien diminue graduellement avec le temps.

Un père refuse l'offre de la fratrie voulant garder l'adulte ayant une DI, alléguant une responsabilité trop lourde. D'autres auteurs constatent aussi ce refus (Bernard \& Goupil, 2012; Joffres, 2002). Taggart et al. (2012) expliquent que malgré des ententes avec la fratrie, les parents doutent de la permanence de ces arrangements, car des circonstances, telle la naissance d'un enfant, peuvent les modifier. Des participants rapportent d'ailleurs ne pas être certains que les fratries connaissent leur futur rôle. Villeneuve et al. (2012) notent aussi que des fratries ignorent tout simplement les attentes parentales. L'étude de Villeneuve montre aussi que la moitié de leurs fratries sont tuteurs ou responsables de la fiducie de l'adulte ayant une DI.

Certains pères disent se sentir coupables envers la fratrie, par exemple, pour leur avoir donné moins d'attention qu'au frère ou à la sœur ayant une DI. Ceci expliquerait, du moins en partie, pourquoi les participants ont de la réticence à demander l'aide des fratries (Villeneuve et al., 2012). Les pères voudraient à la fois le bonheur de la personne ayant une DI et celui de la fratrie, ne souhaitant pas engager cette dernière par la responsabilité d'un soutien à long terme.

\section{Communications avec la personne ayant une DI sur son avenir}

La convention des Nations Unies (2006) sur les droits des personnes handicapées reconnaît le droit à ces personnes de faire des choix. Or, moins de la moitié des participants ont discuté avec la personne ayant une DI de ses préférences pour l'après-parents. La déficience intellectuelle qu'elle soit moyenne, sévère ou profonde entraîne des problèmes dans la communication et la compréhension de certains concepts tels l'avenir ou le vieillissement (Dusart, 1998). Ces difficultés entravent les discussions sur la transition avec la personne ayant une DI et l'identification des choix de cette dernière. Les familles pourraient-elles bénéficier, pour discuter de l'avenir, d'une communication soutenue par des aides visuelles, tel que le propose Cobigo (2008) pour identifier les préférences socioprofessionnelles des personnes ayant une DI? Curryer, Stancliffe et Dew (2015) soulignent aussi les dilemmes des familles entre leur désir de protection et celui de rendre la personne indépendante. Certaines familles, convaincues de connaitre le meilleur choix à poser pour la personne ayant une DI, décideront alors à sa place. Des domaines sensibles génèrent aussi des craintes. Ainsi, Foley (2012) constate que si plusieurs adultes ayant un syndrome de Down travaillent, certains parents restreignent les choix de ces adultes, entre autres, concernant la sexualité.

Le vieillissement des parents, leur perte d'autonomie et l'anticipation de leur propre décès s'associent au départ de la personne ayant une DI. Ces sujets entravent possiblement la mise en place d'apprentissages favorisant l'autodétermination ou le départ de la résidence familiale de la personne.

Les adultes ayant une DI bénéficient de s'exprimer sur les transitions et leur planification (Martin, O'Connor-Fenelon, \& Lyons, 2012; Shaw et al., 2011). Le gouvernement de l'Ontario (Ministère des Services sociaux et communautaires, 2013) a d'ailleurs présenté un guide de planification autodirigée qui promeut le rôle de la personne ayant une DI, même si cette personne utilise une communication surtout non verbale. Ce guide propose plusieurs méthodes utilisées avec l'aide d'un facilitateur ou par les aidants naturels: le MAPS, PARCOURS, Essential Lifestyle Planning et d'autres démarches.

\section{Apports et limites de l'étude}

Cette étude dégage les actions et les propos des pères sur le choix de la cohabitation, les contraintes associées à ce mode de vie, leurs rêves ou leurs émotions face à l'avenir et l'état de leur planification de la transition de l'après-parents. L'utilisation conjointe de questions fermées et ouvertes permet de tracer un premier portrait, certes exploratoire, de la situation. Ainsi, ces données indiquent que la majorité des pères ne sont pas engagés dans des démarches avancées de planification. De même, un faible nombre de pères semblent impliquer activement la personne ayant une DI dans des discussions, avec des exemples concrets pour soutenir sa compréhension sur la transition de l'après-parents, tel que le suggère le ministère des Services sociaux et communautaires de l'Ontario (2013). Cette étude présente toutefois plusieurs limites. Si l'utilisation de questions fermées permet d'identifier des informations précises sur la transition, elle diminue probablement la profondeur des entretiens et l'expression des émotions. Le nombre de participants et de régions du Québec d'où ils proviennent étant peu élevé, une étude à plus large échelle s'avère nécessaire. De plus, les participants vivent tous en couple. Une étude auprès de familles monoparentales pourrait apporter d'autres données utiles à la planification des services. 


\section{Recommandations}

Les pères et les mères vieillissants ont aussi des besoins. Leur rôle d'aidants naturels entraine des conséquences sur leur santé mentale et physique (Ouellette-Kuntz et al. 2006). Eley, Boyes, Young et Hegney (2009) précisent ainsi que le vieillissement parallèle de la personne ayant une DI et de ses parents peut entrainer une double crise où à la fois la situation de l'aidant et celle de la personne ayant une DI deviennent critiques. Il importe donc d'offrir des moments de répit aux parents qui n'ont, entre autres, que peu de moments pour se retrouver en couple. Des actions doivent être mises en place pour aider ces familles dans un contexte où les personnes ainées nécessitent massivement des services résidentiels et présentent de nouveaux besoins associés au vieillissement.

Les démarches de planification de la transition devant être amorcées tôt, un moment précis ne devrait-il pas être identifié par les centres de réadaptation en déficience intellectuelle pour traiter de cette question? Il y aurait alors lieu d'évaluer les meilleures méthodes pour communiquer avec les parents. Ceci représente un défi compte tenu du roulement de personnel qui a cours aujourd'hui et de la relation de confiance nécessaire pour traiter de cette question sensible. De plus, les intervenants ne peuvent pas compter sur l'initiative de tous les parents pour initier cette planification, car plusieurs parents vivent des émotions négatives face à l'avenir et certains ont connu des expériences jugées sans résultats avec certains services scolaires ou de réadaptation. Il s'agit d'une situation complexe pour les intervenants, où s'entremêlent l'expérience passée des parents, leurs émotions en regard de la séparation, l'incertitude face à l'avenir et la diminution avec l'âge de leurs propres capacités. Toute cette complexité ne devrait-elle pas faire l'objet de recherches futures et de formation pour le personnel impliqué dans la planification des transitions de l'après-parents?

Une information claire et centralisée (un site web, par exemple) et accessible à tous les parents pourrait informer sur la transition de l'après-parents et les services (d'éducation, d'emploi, de santé, de services résidentiels, de répit, etc.) disponibles pour les adultes. Des outils de planification pour des personnes ayant une DI, entre autres, pour celles ayant des problèmes de communication, pourraient être développés pour permettre une participation active dans cette transition.

\section{Conclusion}

Tout comme les études auprès des couples ou des mères, notre étude révèle le peu de planification des pères en regard de l'après-parents. Qui sera responsable de l'adulte en situation de handicap lorsque le parent ne sera plus là? Cette question de fond préoccupe l'ensemble des familles vivant avec une personne ayant une DI. Comme dans l'étude de Taggart et al. (2012), plusieurs de nos participants ont contenu leurs larmes lors de l'entrevue. Ces émotions témoignent de l'importance de cette transition et du besoin de soutien des familles, même si ces dernières repoussent la planification de l'après-parents au dernier moment.

Nos résultats indiquent que les pères d'une personne ayant une DI vieillissent dans une situation différente des parents qui n'ont pas les responsabilités du soutien d'une personne ayant une DI. Même à la retraite, ils assument quotidiennement un rôle majeur d'aidant naturel. La localisation des services (centres de réadaptation, milieux de travail adaptés, spécialistes, etc.) conditionne aussi pour certains pères leur propre lieu de résidence. Alors que dans la majorité des familles, au départ des enfants du domicile familial, le couple retrouve une intimité, cette situation est absente pour ces pères vieillissants. Notre étude souligne les besoins de ces pères vieillissants pour planifier l'aprèsparents. Elle fait état du besoin de répit de ces pères et des émotions intenses qui accompagnent l'aprèsparents. Dans ce contexte, des recherches ultérieures pourraient évaluer les modalités de soutien qu'il serait souhaitable d'offrir à ces pères : groupes de rencontre en personne ou en ligne, informations sur la transition, intervenants spécialisés sur cette question, services de répit prenant en compte l'âge et la santé des parents, etc. Le moment de la transition générant aussi de nombreuses inquiétudes, il y aurait lieu d'évaluer comment accompagner les parents dans ce moment crucial pour eux et la personne qui présente une DI. L'étude d'une transition graduelle (où la personne fréquente à la fois le foyer familial et un nouveau lieu de vie) pourrait aussi faire l'objet de différentes recherches.

Notre étude révèle aussi que la personne elle-même n'est que peu impliquée dans la planification de l'après-parents. Aussi, il serait intéressant de mener d'autres études auprès des personnes ayant une DI en prenant en compte leur niveau de déficience et le rôle joué auprès de leurs parents vieillissants.

\section{References}

Azéma, B., \& Martinez, N. (2005). Les personnes handicapées vieillissantes: espérances de vie et de santé, qualité de vie. Revue française des affaires sociales, 2, 297-333.

Bernard, M.-A. \& Goupil, G. (2012). L'après-parents: étude exploratoire sur les perceptions de mères qui vieillissent avec un adulte ayant une déficience intellectuelle. $\mathrm{La}$ Revue canadienne du vieillissement, 31, 65-72. doi: 10.1017/ S0714980811000602

Bernard, M.-A. (2011). Transition en cours de vie de personnes ayant une déficience intellectuelle: recherche 
exploratoire sur l'après-parents. Essai doctoral non publié, Département de psychologie, Université du Québec à Montréal, Montréal, Canada.

Bigby, C. (1996). Transferring responsibility: The nature and effectiveness of parental planning for the future of adults with intellectual disability who remain at home until mid-life. Journal of Intellectual and Developmental Disability, 21, 295-312.

Bigby, C., Webber, R. W. \& Bowers, B. (2015). Sibling roles in the lives of older group home residents with intellectual disability: Working with staff to safeguard wellbeing. Australian Social Work, 68, 453-468. doi: 10.1080/0312407X.2014.950678

Bittles, A. H., Petterson, B. A., Sullivan, S. G., Hussain, R., Glasson, E. J. \& Montgomery, P. D. (2002). The influence of intellectual disability on life expectancy. Journal of Gerontology Biological and Medical Sciences, 57, 470-472.

Burke, M. M., \& Heller, T. (2016). Disparities in unmet service needs among adults with intellectual and other developmental disabilities. Journal of Applied Research in Intellectual Disabilities, 30, 898-910. doi: /abs/10.111/jar122821 Disabilities, 30

Cobigo, V. (2008). Élaboration d'une méthode d'évaluation des préférences liées à des activités socioprofessionnelles de personnes présentant une déficience intellectuelle dont le mode de communication est non verbal [Thèse de doctorat]. Université du Québec à Montréal, Montréal, QC.

Curryer, B., Stancliffe, R. J. \& Dew, A. (2015). Self-determination: Adults with intellectual disability and their family. Journal of Intellectual Developmental Disability, 40, 394-399. doi: 10.3109/13668250.2015.1029883

Davys, D., Mitchell, D. \& Haigh, C. (2010). Futures planning, parental expectations and sibling concern for people who have a learning disability. Journal of Intellectual Disabilities, 14, 167-183. doi:10.1177/1744629510385625

Davys, D., Mitchell, D. \& Martin, R. (2017). Fathers of people with intellectual disability: A review of the literature. Journal of Intellectual Disabilities, 2, 175-196. doi: $10.1177 / 1744629516650129$

Dusart, A. (1998). Les personnes déficientes intellectuelles face à la mort: retentissement de la perte d'un proche et attitude de l'entourage. Paris, France: Fondation de France.

Eley, D., Boyes, J., Young, L. \& Hegney, D. (2009). Adults with intellectual disability in regional Australia: Incidence of disability and provision of accommodation support to their ageing carers. Australian Journal of Rural Health, 17, 161-166. doi: 10.1111/j.1440-1584.2009.01062.x

Foley, S. (2012). Reluctant 'Jailors' speak out: Parents of adults with Down syndrome living in the parental home on how they negotiate the tension between empowering and protecting their intellectually disabled sons and daughters. British Journal of Learning Disabilities, 41, 304-311. doi: 10:1111/j.1468-3156.2012.00758x
Goupil, G. (2004). Plans d'intervention, de services et de transition. Montréal, QC: Gaëtan Morin Éditeur Ltée.

Gouvernement du Canada (2004). Vieillir avec une déficience intellectuelle. Ottawa, ON: Ministre de Travaux publics et Services gouvernementaux Canada.

Grey, J. M., Griffith, G. M., Totsika, V. \& Hastings, R. P. (2015). Families' experiences of seeking out-of-home accommodation for their adult child with an intellectual disability. Journal of Policy and Practice in Intellectual Disabilities, 12,47-57. doi:10.1111/jppi.12106

Heller, T. \& Kramer, J. (2009). Involvement of adult siblings of persons with developmental disabilities in future planning. Intellectual and Developmental Disabilities, 47(3), 208-219. doi:10.1352/1934-9556-47.3.208

Hole, R. D., Stainton, T. \& Wilson, L. (2013). Ageing adults with intellectual disabilities: Self-advocates' and family members' perspectives about the future. Australian Social Work, 66, 571-589. doi:10.1080/031240 7X.2012.689307

Institut de la statistique du Québec (2015). La mortalité et l'espérance de vie au Québec en 2014. Québec, QC. Récupéré de http://www.stat.gouv.qc.ca/statistiques/populationdemographie/bulletins/coupdoeil-no40.pdf

Joffres, C. (2002). Barriers to residential planning: Perspectives from selected older parents caring for adult offspring with lifelong disabilities. Canadian Journal on Aging, 21, 303-311. doi:10.1017/S0714980800001550

Krauss, M. W. \& Seltzer, M. M. (1998). Life course perspectives on mental retardation research: The case of family caregiving. In J. A. Burack, R. M. Hodapp \& E. Zigler (Eds.), Handbook on mental retardation and development (Cambridge, England: Cambridge University Press), (pp. 504-520).

Lacasse-Bédard, J. (2009). Dimensions subjectives de l'expérience de planification d'arrangements de vie chez les parents perpétuels vieillissants vivant avec un adulte présentant un syndrome de Down [Mémoire de maitrise]. Récupéré de http:/ / www. collectionscanada.gc.ca/obj/thesescanada/vol2/002/ MR61471.PDF

Longtin, V. (2015). Étude exploratoire des transitions de milieux de vie en communauté des personnes présentant une déficience intellectuelle [Thèse de doctorat]. Récupéré de https: / / papyrus.bib.umontreal.ca/xmlui/handle/1866/16035

Martin, A.-M., O'Connor-Fenelon, M. \& Lyons, R. (2012). Non-verbal communication between Registered Nurses Intellectual Disability and people with an intellectual disability: An exploratory study of the nurse's experiences. Journal of Intellectual Disabilities, 16, 61-75. doi: $10.1177 / 1744629512440938$

Maulik, P. K., Mascarenhas, M. N., Mathers, C. D., Dua, T. \& Saxena, S. (2011). Prevalence of intellectual disability: A meta-analysis of population-based studies. Research in Developmental Disabilities, 32, 419-436. doi:10.1016/j. ridd.2010.12.018 
Miles, M. B. \& Huberman, A. M. (2003). Analyse des données qualitatives. Bruxelles, Belgique: De Boeck Supérieur.

Ministère des Services sociaux et communautaires (2013). Guide de planification autodirigée et de facilitation. Toronto, ON. Récupéré de http://www.mcss.gov.on.ca/documents/ $\mathrm{fr} / \mathrm{mcss} /$ publications / developmental/Person_DirectedPlanning.pdf

Nations Unies (2006). Convention relative aux droits des personnes handicapées. New York, NY: Auteur. Récupéré de http:/ / www.un.org/disabilities/documents/convention/convoptprot-f.pdf

Ouellette-Kuntz, H., Minnes, P., Garcin, N., Martin, C., Lewis, S.M.E. \& Holden, J. J. A. (2005). Addressing health disparities through promoting equity for individuals with intellectual disability. Revue canadienne de santé publique, 96(2), 8-22.

Ouellette-Kuntz, H., Coo, H., Yu, C. T., Chudley, A. E., Noonan, A., Breitenbach, M., Ramji, N., Prosick, T., Bedard, A. \& Holden, J. J. (2006). Prevalence of pervasive developmental disorders in two Canadian provinces. Journal of Policy and Practice in Intellectual Disabilities, 3(3), 164-172.

Rioux, S. \& St-Pierre, M. (2013). Le vieillissement des personnes qui présentent une déficience intellectuelle. Montréal, QC: Comité régional des associations pour la déficience intellectuelle.

Robinson, S., Weiss, J. A., Lunsky, Y. \& Ouellette-Kuntz, H. (2015). Informal support and burden among parents of adults with intellectual and/or developmental disabilities. Journal of Applied Research in Intellectual Disabilities, 29, 1-10. doi:10.1111/jar.12184

Salvatori, P., Tremblay, M. \& Tryssenaar, J. (2003). Living and aging with developmental disability: Perspectives of individuals, family members and service providers. Journal of Developmental Disabilities, 10, 1-20. Récupéré de http:/ / www.opadd.on.ca / News / documents/paperagingwddsalvatorietal.pdf

Schalock, R. L., Borthwick-Duffy, S., Bradley, V. J., Buntinx, W. E. M., Coulter, D. L., Craig, E. M., Gomez, S. C., Lachapelle, Y., Luckasson, R., Reeve, A., Shogren, K. A., Snell, M. E., Spreat, S., Tassé, M. J., Thompson, J. R.,
Verdugo-Alonso, M. A., Wehmeyer, M. L. \& Yeager, M. H. (2011). Déficience intellectuelle: définition, classification et systèmes de soutien (11e éd.) [trad. sous la direction: D. Morin]. Trois-Rivières, QC: Consortium national de recherche sur l'intégration sociale (Ouvrage original publié en 2011).

Seltzer, M. M., Floyd, F., Song, J., Greenberg, J. \& Hong, J. (2011). Midlife and aging parents of adults with intellectual and developmental disabilities: Impacts of lifelong parenting. American Association on Intellectual and Developmental Disabilities, 116, 479-499. doi:10.1352/1944-7558-116.6.479

Shaw, K., Cartwright, C. \& Craig, J. (2011). The housing and support needs of people with an intellectual disability into older age. Journal of Intellectual Disability Research, 55, 895-903. doi:10.1111/j.1365-2788.2011. 01449.x

Statistique Canada (2010). Enquête sur la participation et les limitations d'activités de 2006: l'expérience de travail des personnes avec incapacités au Canada. Récupéré de http: / / www.statcan.gc.ca / pub / 89-628-x/89-628x2008007-fra.htm

Taggart, L., Truesdale-Kennedy, M, Ryan, A. \& McConkey, R. (2012). Examining the support needs of ageing family carers in developing future plans for a relative with an intellectual disability. Journal on Intellectual Disabilities, 13, 217-234. doi:10.1177/1744629512456465

Villeneuve, M., Goupil, G, \& Gascon, H. (2012). Perceptions des frères et des sœurs adultes d'une personne présentant une déficience intellectuelle sur leurs relations mutuelles dans la famille, leurs projets de vie et le soutien souhaité. Revue francophone de la déficience intellectuelle, 23, 104-120. doi:10.7202/1015666ar

Wolfensberger, W. (2011). Twenty predictions about the future of residential services in mental retardation. Intellectual and Developmental Disabilities, 49, 411-415. doi:10.1352/1934-9556-49.6.411

Woodman, A. C., Mailick, M. R., Anderson, K. A. \& Esbensen, A. J. (2014). Residential transitions among adults with intellectual disability across 20 years. American Journal on Intellectual and Developmental Disabilities, 119, 496-515. doi:10.1352/1944-7558-119.6.496 